\title{
Pierre Mounier \\ Die Werkstatt öffnen: Geschichtsschreibung in Blogs und Sozialen Medien
}

Aus dem Französischen übersetzt von Inger Brandt und Mareike König.

In welcher Weise verändern die digitalen Technologien die Arbeitsbedingungen für Historikerinnen und Historiker? Bereits seit mehreren Jahrzehnten gibt es darauf Antworten. Die Erstellung quantitativer Datenbanken, die Digitalisierung wichtiger Quellen, die kartografische Darstellung und die Analyse sozialer Netzwerke mit IT-Werkzeugen sind dabei die ältesten und wichtigsten Meilensteine. Die Retrodigitalisierung und Online-Veröffentlichung akademischer Literatur der Disziplin, seien es Zeitschriften oder Bücher, stellen eine weitere Etappe in dieser Richtung dar. Die digitale Technologie hat bis heute zugleich den Werkzeugkasten der historisch Arbeitenden - um den schönen Ausdruck aus dem gleichnamigen Blog „La Boite à Outil des Historiens“ (www. boiteaoutils.info/) aufzugreifen - und ihre Publikationsmöglichkeiten grundlegend verändert.

Einige berühmte Beispiele aus der Vergangenheit haben die Geschichtswissenschaft gelehrt, dass nicht immer eindeutig bestimmt werden kann, wann es Zeit ist, die „Revolution zu beenden“. Revolutionäre Phänomene nähren sich mitunter selbst. Man kann daher von einer "permanenten Revolution" sprechen, wie es der Soziologe Philippe Breton mit Blick auf das Internet tut (Breton 2000). Es sind heute also die neuen Werkzeuge und vor allem die neuen Praktiken der vernetzten Kommunikation, die - nachdem sie seit einigen Jahren immer stärker von der breiten Öffentlichkeit genutzt wurden - seit kurzem auch in den Sozial- und Geisteswissenschaften auftauchen. Es handelt sich um die Werkzeuge des Web 2.0, die sich von der Vorgängergeneration durch ihre einfache Nutzung und durch ihren gleichzeitig unkonzentrierten, horizontalen und unmittelbaren Charakter der Kommunikationspraktiken unterscheiden: Plattformen für content sharing (Dokumente, Fotos, bibliografische Referenzen), Blogs und Soziale Netzwerke stellen ein Ensemble an „sozialen Medien“ dar. Deren Anwendung durch Historikerinnen und Historiker wird zunehmend selbst Gegenstand der Debatte. Das zeigt beispielsweise das 2011 vom Deutschen Historischen Institut Paris organisierte Kolloquium „Im Netz der sozialen Medien - Neue Publikations- und Kommunikationswege in den Geisteswissenschaften" (Mounier 2011). Es war besonders interessant, im Verlauf des Kolloquiums Wissenschaftlerinnen und Wissenschaftler unterschiedlicher 
Herkunft darüber reden zu hören, wie sie sich die neuen Werkzeuge aneignen, um ihre Kommunikationspraktiken von Grund auf zu erneuern. Man traf dort Historiker wie Peter Haber von der Universität Basel mit seinem Blog bei hist.net (weblog.histnet.ch/), André Gunthert von der EHESS, der die Plattform „Culture visuelle“ (culturevisuelle.org/) für wissenschaftliche Blogs über visuelle Geschichte aufgebaut hat, oder auch Klaus Graf, der sein bekanntes Weblog "Archivalia“ (archiv.twoday.net/) vorstellte und mit der provokanten These „ein Wissenschaftler ohne Blog ist ein schlechter Wissenschaftler“ für Aufsehen sorgte (König 2011).

\section{Eine Arbeit bei "geöffneter Werkstatt"}

Trotz der Polemik kann es interessant sein nachzuvollziehen, welche Rolle ein Wissenschaftsblog bei der Arbeit der Forschenden spielen kann und ab welchem Moment es im Forschungsprozess eingesetzt wird. Und da es sich offensichtlich um ein Kommunikationsmittel handelt, stellt sich die Frage nach der Einordnung von Blogs im Vergleich zu den traditionellen Publikationen wie Zeitschriften und Büchern, die mittlerweile auch im Internet vertrieben werden. Bei aufmerksamer Betrachtung wissenschaftlicher Blogs wie den gerade erwähnten oder den auf der von OpenEdition in Frankreich betriebenen Plattform hypotheses.org (hypotheses.org/) gehosteten Blogs kristallisiert sich folgendes Hauptmerkmal heraus (zu den Geschichtsblogs bei hypotheses.org siehe auch den Beitrag von Mareike König in diesem Band): Während die traditionellen Publikationen scharf die interne Kommunikation (Forschungsergebnisse adressiert an Kolleginnen und Kollegen) von der externen Kommunikation (an die breite Öffentlichkeit gerichtete populärwissenschaftliche Aufbereitung) trennen, neigen Wissenschaftsblogs dazu, beide Bereiche an einem Veröffentlichungsort zusammenzuführen. In ihren Blogs arbeiten Historikerinnen und Historiker bei „geöffneter Werkstatt“; sie enthüllen darin ihre tagtägliche Arbeitsroutine, ihre Lektüren und manchmal auch ihre Erkenntnisse, ihre Hypothesen, ihre Zweifel. Damit legen sie einen Aspekt ihrer Forschung offen, noch „während diese im Entstehen“ ist, wie es bei den Soziologen aus der Schule von Bruno Latour heißt (Blanchard 2008). Das weckt sowohl das Interesse der unmittelbaren Kolleginnen und Kollegen, die schnell Zugang $\mathrm{zu}$ dieser Information erhalten und diese eventuell nach wissenschaftlichen Gepflogenheiten kritisieren möchten, als auch das Interesse einer breiteren Öffentlichkeit, die aus unterschiedlichen Gründen am jeweiligen Thema interessiert ist. Wie dem auch sei, eines unterscheidet das Blog deutlich von den kanonischen Publikationsformen, die sowohl in der Form als auch im Erschei- 
nungsrhythmus festgelegt sind: die Freiheit, die Autorinnen und Autoren im Hinblick auf Tonfall, Form, Länge, Thema und Publikationsrhythmus haben. Diese große Freiheit der Ausdrucksmöglichkeiten macht den Wissenschaftlerinnen und Wissenschaftlern Spaß und motiviert sie daher zum Schreiben. Gleichzeitig zieht sie eine hybride Leserschaft an, die mit den Autorinnen und Autoren ein Gespräch beginnen kann. Das Ergebnis ist erstaunlich: Die wissenschaftlichen Blogs unterscheiden sich stark voneinander; die Forschenden, ob allein oder gemeinsam in einem Forschungsteam, veröffentlichen hier alle Arten von Informationen in unterschiedlichen Rhythmen und in variierender Länge. Im Vergleich zu Zeitschriften und anderen konzeptionell stärker eingeschränkten Publikationsformen geben Blogs ein völlig uneinheitliches Bild ab. Sie zeigen das „Sammelsurium des Wissenschaftlers“, wie es Marin Dacos so treffend ausdrückt (Dacos, Mounier 2011). Und über genau diese Vielfalt begreift man das Wesen der Blogs erst richtig, indem die verschiedenen Arten von Informationen, die auf den Blogs verbreitet werden, genauer betrachtet.

Am häufigsten findet man dort kurze Anmerkungen zu den Neuigkeiten aus dem Fachgebiet des Bloggenden: Ankündigungen über Kolloquien und Studientage, call for papers, Meldungen über Neuerscheinungen oder Ausstellungen. Oftmals begnügen sich die Bloggenden damit, die Texte der Aufrufe, die Programme der Kolloquien, den Klappentext des erschienenen Werks wiederzugeben, ohne einen besonderen Kommentar hinzuzufügen. Dies ist beispielsweise beim Blog „Emma“ (emma.hypotheses.org/) der Fall, das aus dem von Damien Boquet und Piroska Nagy geleiteten Forschungsprogramm zu Emotionen im Mittelalter hervorgegangen ist. Man kann vielleicht nach dem Nutzen eines solchen Blogs fragen, das anscheinend nur aus einer Ansammlung von hier und da erschienen Annoncen besteht. In Wirklichkeit handelt es sich um ein wertvolles Monitoring-Tool, da diese Blogs eine manchmal in Kategorien geordnete - qualitative Auswahl an Neuigkeiten zu einem Fachgebiet bieten. Für die gleiche Aufgabe könnten auch andere Tools genutzt werden (wie Anzeige-Services oder Ressourcen-Aggregatoren), aber das Blog ist ein ebenso interessanter Informationsträger. Auf hypotheses.org haben einige Blogs diese Vorgehensweise systematisiert, in dem sie „den Radar eingeschaltet" und "Monitoring-Blogs" eingerichtet haben. So steht beispielsweise "Nuevo Mundo Radar" (nuevomundoradar.hypotheses.org/) für das wissenschaftliche Monitoring des lateinamerikanischen Raums durch das Redaktionsteam der gleichnamigen Zeitschrift. Es sei angemerkt, dass die Praxis des minimalistischen sharing von Meldungen durch die zunehmende Anzahl von unterstützenden Tools verschiedenster Art eine wunderbare Dynamik besitzt. So ist auch das Center for History and New Media in Washington dabei, mit Pressforward (Rosenzweig 2011) ein Tool basierend auf eben diesem Prinzip zu entwickeln. 


\section{Lektürenotizen}

Einige Wissenschaftsbloggende entscheiden sich, alle oder einen Teil ihrer Auswahl zu kommentieren. Dabei handelt es sich dann um richtige Postings in Form von Kurzberichten, wie man sie beispielsweise im 2010 von Raphaëlle Bats gestarteten Blog „Les Préfaces du Griffon“ (gryphe.hypotheses.org/) zur Geschichte des Lyoner Verlagswesens im 16. Jahrhundert findet. Diese knappen Notizen, aufgeschrieben und sogleich im Eifer des Gefechts veröffentlicht, diese ersten Kommentare zu einer Veröffentlichung müssen deutlich unterschieden werden von Buchbesprechungen, die in Wirklichkeit eigene Artikel sind und in Zeitschriften veröffentlicht werden. Die verschiedenen Formen decken sich nicht, sondern ergänzen sich vielmehr: Der im Blog veröffentlichte Eintrag bietet den Vorteil der Schnelligkeit und der Freiheit im Tonfall (häufig ist man dort bissiger), während eine Buchbesprechung formal und inhaltlich überprüft wird, und somit oft umfassender und sachlicher ist. Es ist nicht ausgeschlossen, dass das Eine in gewisser Weise der Entwurf für das Andere wird. Beim Blogbeitrag handelt sich dann um eine Art vorbereitende Stufe, die zu einer peer-review-Veröffentlichung führen kann. Ein Beispiel dafür ist dieser Artikel selbst, der im September 2011 zunächst auf Französisch im Blog „Homo Numericus“ (Mounier 2011) erschienen ist, 2012 dann in der Zeitschrift „Revue d'histoire moderne et contemporaine“. Ein anderes Beispiel ist das Blog „Histoire et culture dans la Tunisie contemporaine“ (hctc.hypotheses.org/) von Kmar Bendana über die tunesische Revolution, deren Beiträge 2012 sogar zusammengefasst als Buch veröffentlicht wurden (Bendana 2012).

Andere Wissenschaftsblogs bieten ausgearbeitete Notizen, die zwar dem Texttyp entsprechend kurz sind, aber über einen bestimmten Forschungsaspekt Bericht erstatten. Hier wäre „Rwanda“ (rwanda.hypotheses.org/) zu erwähnen, ein vielversprechendes Blog, in dem der Doktorand Rémi Korman von seinen Recherchen in den Archiven Ruandas zur Geschichte des Genozids und dem Gedenken daran berichtet. Er erzählt in seinen Notizen relativ sachlich vom Zustand der Quellen, die er bearbeitet, und zwar während er seine Forschungen durchführt. Es ist einer der Vorteile dieser Art von OnlinePublikation, die auf einer sehr simplen Blogtechnik beruht, dass sie von überall und selbst bei schwieriger Internetverbindung durchgeführt werden kann. Andere Forschergruppen, wie das Team, das sich im Blog "Terriat“ (terriat. hypotheses.org/103) mit „Warteräumen“ (territoires de l'attente) im Migrationsprozess in den amerikanischen und atlantischen Gesellschaften befasst, entscheiden sich eher für kurze Notizen, die historische Quellen wie Fotos und Interviewausschnitte kommentieren. Hier wird das Schriftstück weder durch einen umfassenden Anmerkungsapparat noch durch einen Zweifel an 
der Kohärenz oder der Konstruktion gestört. Es handelt sich um Notizen, die Tag für Tag in dieser Form im Blog veröffentlicht werden. Im Gegensatz zur Zeitschrift, in der die Artikel lektoriert und von Fachkolleginnen und Fachkollegen überprüft werden, handelt es sich beim Blog ausschließlich um das Forschungsjournal einer Wissenschaftlerin oder eines Wissenschaftlers - und um nichts weiter. Aber als solches stellt es eine unschätzbare Quelle für Informationen aus erster Hand dar. Das Blog kann auch kollektiv organisiert sein, was den Charakter von "Aimos" (aimos.hypotheses.org/) widerspiegelt. Dort haben sich einige junge Forschende zusammengefunden, die untereinander, aber auch mit einer breiteren Öffentlichkeit, Ressourcen über die Kunst und Bilder der Arbeiter- und Sozialbewegung austauschen wollen.

Das Blog besitzt darüber hinaus gelegentlich in sich selbst einen historischen Wert, was das Team vom Centre d'étude des mondes africains deutlich erkannt hat. Es veröffentlicht in "Les Cahiers de Terrain de Raymond Mauny“ (mauny. hypotheses.org/) online eine digitalisierte Version der Aufzeichnungen des bekannten Archäologen. Dabei erfolgt die Publikation der einzelnen Aufzeichnungen im gleichen Zeitabstand, in dem sie der Forscher damals notierte ... nur 60 Jahre später. Abgesehen von der Neugier, die eine solche Aktion weckt, und dem Geschick, mit dem dieser Teil des wissenschaftlichen Erbes gewürdigt wird, kann man nur erstaunt sein über die historische Kontinuität einer wissenschaftlichen Tradition, die sich so von einem Informationsträger zum nächsten überträgt. Und jenseits der revolutionären Bejahung, die die Entwicklung digitaler Technologien oftmals begleiten: das Wissenschaftsblog oder das Online-Forschungsjournal ist nichts anderes als ein gemeinsam geführtes Ausgrabungsjournal, was gleichzeitig alles und nichts verändert. Im Übrigen wollte Christian Jacob genau das im zweiten Band seiner von ihm herausgegebenen meisterhaften historischen Untersuchung über die "Lieux de Savoirs“ (Jacob 2011) erreichen, indem er ein Kapitel über wissenschaftliche Blogs einforderte.

\section{Eine schizophrene Kommunikation?}

Über das Ausgrabungsjournal hinaus entscheiden sich einige Forscherteams, ihre Beiträge stärker zu edieren, indem sie Textstile und Präsentation der Informationen vereinheitlichen. Dies trifft beispielsweise auf das „Blog de la Grotte des Fraux" (champslibres.hypotheses.org/) zu, das sich mit den Ausgrabungen in der gleichnamigen Höhle im Departement Dordogne befasst. Dieses reich bebilderte Blog berichtet äußerst gewissenhaft vom Fortschritt der Arbeiten in 
den Tiefen der Grotte. Ein interessanter Fall, da es zugleich eine fachlich sehr spezialisierte Leserschaft (die Texte sind oft sehr technisch) und ein breites $\mathrm{Pu}-$ blikum von Liebhaberinnen und Liebhabern der Archäologie anspricht:

„Die spezifische Welt der Kunst und der Höhle, mit all den Bildern, Vorstellungswelten und dem Verbotenen, das damit verbunden ist, verkörpert die Schwierigkeiten, mit einem speziellen Forschungsgegenstand konfrontiert zu sein. Da sowohl die wissenschaftliche Gemeinschaft wie auch die Öffentlichkeit nur schwer Zugang zu diesen physischen und geistigen Orten finden, ist es unser Anliegen, den Forschenden und der Öffentlichkeit diese Gedankenelemente zur Verfügung zu stellen.

Diese Haltung mag schizophren erscheinen! Wie soll die korrekte Durchführung eines Forschungsvorhabens - das ein Abwägen der Hypothesen, eine Prüfung der Protokolle und eine Bestätigung der Ergebnisse erfordert - mit der Veröffentlichung von vorläufigen Rohdaten, die Gefahr laufen widerlegt zu werden, zusammengeführt werden? Das ist die große Herausforderung in diesem Forschungsblog: möglichst schnelle Verbreitung der Daten auf einem wissenschaftlichen Portal, bei gleichzeitiger Wahrung der Freiheit, sich widersprechen und sein Urteil ändern zu können. Das ist die Idee, die uns an diesem Projekt so fasziniert hat."

Schizophren gestaltet sich das Projekt „Blog de la Grotte des Fraux“ allerdings keineswegs, ganz im Gegenteil. Denn es wird versucht, im selben editorischen Rahmen zugleich nicht belegte Arbeitshypothesen, bestätigte Ergebnisse und Erläuterungen für ein fachfremdes Publikum zu vereinen. Aus diesem Grund stellt das Blog eines der interessantesten und innovativsten Experimente auf dem Gebiet der wissenschaftlichen Kommunikation dar.

Von einem Blog mit Textentwürfen bis zum stärker ausgearbeiteten Grabungsjournal hat das Online-Forschungsjournal den Vorteil einer großen Flexibilität, die es Wissenschaftlerinnen und Wissenschaftlern erlaubt, die Art und Weise der Nutzung des eigenen Blogs selbst festzulegen. Die Distanz zum Blog ist nicht leicht aufzubauen und wird in tausend aufeinanderfolgenden Anpassungen vorgenommen, wie Benoît Kermoal es in einem Beitrag seines Blog „Enklask/Enquête“ (enklask.hypotheses.org/) ausdrückt, bei dem es wie in seiner gleichnamigen Doktorarbeit um die Geschichte der sozialistischen Bewegung in der Bretagne geht.

„Trotz meiner Vorsicht scheinen einige Leser zu glauben, dass die Notizen in diesem Blog meine gesamte Forschungsarbeit ausmachen. Es handelt sich aber lediglich um Skizzen, um Teile eines Puzzles ohne Vorlage, um eine Ansammlung von Legosteinen, die schlecht zusammengefügt sind und noch kein Objekt bilden. Ich mache aber dennoch weiter, und zwar vor allem, um mich im Schreiben zu üben. Ich betreibe diese Form der Übung aber auch deshalb weiter, weil sie mir erlaubt, meine Forschung zu ordnen, zu klassifizieren, gedankliche Pfade 
zu kreuzen und zu verfolgen und nicht den lähmenden Eindruck zu bekommen, auf der Stelle zu treten.

Das kann dem Leser natürlich als Entwurf erscheinen, als zu skizzenhaft und zweifellos auch als ähnlich egozentrisch wie ein Forschungsjournal, das man für sich behält. Aber „Enklask/Enquête“ ist kein Notizheft mit Textentwürfen (davon habe ich bereits genug); es ist auch keine Aneinanderreihung von Beiträgen, die zusammen fertige und redigierte Artikel nach den Regeln der Geschichtskunst bilden. Es handelt sich um eine hybride Form, eine Erforschung mehrerer Wege, bevor der richtige gefunden ist. Man sollte sich nicht davor fürchten, wieder zurück zu gehen, sich einzugestehen, dass man sich verlaufen hat oder seine Fehler anzuerkennen. Das Wichtige ist jedoch, mit seinen Forschungen voranzukommen. Eine solche hybride Form impliziert zusätzlich eine Vielzahl von Änderungen im Ton, in der Sichtweise und im „Ansatzpunkt“ (Kermoal 2011).

\section{Die Archive öffnen?}

Der Bereich der Archive spielt eine zentrale Rolle in der Werkstatt der Historikerinnen und Historiker. Hier tun sie sich schwer mit der Preisgabe von Informationen. Die Entdeckungen, die sie dort machen können, könnten ihnen möglicherweise einen Wettbewerbsvorteil gegenüber den Kolleginnen und Kollegen verschaffen. Obwohl es für Historikerinnen und Historiker oft schwierig ist, Informationen aus „ihren“ Archiven öffentlich zu machen, insbesondere solange die eigene Arbeit bisher noch nicht in einem oder mehreren Werken veröffentlicht wurde, haben einige Wissenschaftlerinnen und Wissenschaftler dennoch beschlossen, bei „offenem Archiv“ zu arbeiten. Ein solcher Fall ist Isabelle Brancourt, die seit mehreren Jahren über das „Parlament von Paris"(parlementdeparis.hypotheses.org/) forscht. In ihrem Blog betreibt sie vor allem „die Bereitstellung [ihres] persönlichen Forschungsjournals mit der Analyse der Sammlung des Gerichtsschreibers Jean-Gilbert Delisle, im Dienste des zivilen Urkundsbeamten des Pariser Parlaments in der ersten Hälfte des 18. Jahrhunderts" (Brancourt 2010). Es sind nicht viele Beispiele bekannt, bei denen Wissenschaftlerinnen und Wissenschaftlern ihre Rohdaten während des laufenden Forschungsprozesses zur Verfügung stellen. Da diese Vorgehensweise anscheinend sehr selten ist, soll sie hier besonders hervorgehoben werden soll, denn sie hinterfragt die gängigste Gewohnheit der Disziplin.

Es wäre schade, diesen Überblick der verschiedenen Nutzungsmöglichkeiten von wissenschaftlichen Blogs abzuschließen, ohne die methodologischen Blogs zu erwähnen, die in mehreren Disziplinen entstehen. Dazu gehören 
zum Beispiel in der Soziologie "Quanti“ (quanti.hypotheses.org/), aber auch in der Geschichtswissenschaft „Devenir Historien-ne“ (devhist.hypotheses. org/), oder interdisziplinär das Blog „Les Aspects concrets de la Thèse“ (act. hypotheses.org/), das nicht nur Historikerinnen und Historiker, sondern auch andere Disziplinen anspricht. „Thinking with my fingers“ (torillsin.blogspot. fr/): Für die Mehrheit der Bloggenden wie auch für die Wissenschaftlerin Torill Mortensen, die diesen Titel für ihr Blog gewählt hat, handelt es sich beim wissenschaftlichen Blog zunächst um ein Werkzeug zur Selbstreflexion, da das Blog ihnen erlaubt, durch die Erklärung ihrer Forschung in ihrer Arbeit voranzukommen. Jedoch sind Blogs nicht trivial: Online gestellt wird es veröffentlicht und weiter verbreitet. Das bringt eine Mehrdeutigkeit mit sich, die von Benoît Kermoal schön zusammengefasst wurde: „An wen richten sich die Beiträge? Das kommt darauf an: mal an mich selbst, mal an alle Leser, manchmal an Doktoranden oder an Liebhaber der lokalen Geschichte oder der Geschichte der Arbeiterbewegung. Aber es ist vor allem eine Arbeit, die mir erlaubt, meine Forschungen zu strukturieren." (Kermoal 2011). Diese Mehrdeutigkeit ist der Preis für die Freiheit und sie kann mit gutem Recht kritisiert werden. Und das wird sie im Übrigen auch oft. Sie macht aber auch den Reichtum eines wissenschaftlichen Blogs aus. Ein Blog kann Kreativität freisetzen, weil es die Schreibenden von jeglichem akademischen Formalismus befreit. Allerdings kann dieser Prozess nur funktionieren, wenn das Blog der öffentlichen Lektüre unterworfen wird, sei es mit Blick auf unterschiedliche oder unbestimmte Empfängerinnen und Empfänger. Es stellt also für Doktorandinnen und Doktoranden wie auch für erfahrene Wissenschaftlerinnen und Wissenschaftler ein nicht zu leugnendes Risiko dar. Das ist die Sache jedoch wert.

\section{Zitierte Literatur}

Kmar Bendana, Un an et après? in: HCTC, 14.1.2012, hctc.hypotheses.org/103. Antoine Blanchard, Comment montrer la „science en train de se faire“?, in: La Science, La Cité, 31.5.2008, www.enroweb.com/blogsciences/index.php?2008/ $05 / 31 / 261$.

Isabelle Brancourt, Mon „carnet“ Delisle?: Son Journal pour l'année 1730 (I), in: Parlement de Paris (XVIe-XVIIIe siècle), 9.2.2010, parlementdeparis. hypotheses.org/218.

Philippe Breton, Le culte de l'internet: une menace pour le lien social?, Paris (Editions La Découverte) 2000. 
Marin Dacos, Pierre Mounier, Les Carnets de recherches en ligne. Espace d'une conversation scientifique décentrée, in: Lieux de savoir 2. Les mains de l'intellect, Paris 2011, S. 333-353.

Christian Jacob (Hg.), Lieux de savoir 2, Les mains de l'intellect, Paris 2011.

Benoît Kermoal, Seulement la partie visible de l'iceberg, in: Enklask / Enquete, 6.9.2011, enklask.hypotheses.org/257.

Benoît Kermoal, Seulement la partie visible de l'iceberg, in: Enklask / Enquete, 6.9.2011, enklask.hypotheses.org/257.

Mareike König, Tweets und Gedanken zur Tagung „Im Netz der sozialen Medien“, in: Digital Humanities à l'IHA, 11.7.2011, dhiha.hypotheses.org/284.

Pierre Mounier, Dans la toile des médias sociaux / Im Netz der sozialen Medien 27-28 Juin 2011, in: Digital Humanities à l'IHA, 24.5.2011, dhiha.hypotheses. org/25.

Pierre Mounier, Ouvrir l'atelier de l'historien: médias sociaux et carnets de recherche en ligne, in: Homo Numericus, 11.9.2011 www.homo-numericus.net/ spip.php?article304.

Pierre Mounier, Ouvrir l'atelier de l'historien. Médias sociaux et carnets de recherche en ligne, in: Revue d'histoire moderne et contemporaine $\mathrm{n}^{\circ} 58-4$ bis -5 (2012), S. 101-110 cairn.info/revue-d-histoire-moderne-et-contemporaine2011-5-page-101.htm.

Roy Rosenzweig, Introducing PressForward, in: Center for History and New Media, 24.6.2011, chnm.gmu.edu/news/introducing-pressforward/. 
